\title{
Transdermal Delivery of Sumatriptan for the Treatment of Acute Migraine
}

\author{
Mark W. Pierce \\ NuPathe Inc., Conshohocken, Pennsylvania 19428
}

\begin{abstract}
Summary: Migraine is a common, multisymptom disorder that can severely impact the daily activities of migraineurs. Triptans (primarily sumatriptan) are the most commonly prescribed treatment for migraine and are considered a relatively safe and effective initial therapy. Unfortunately, current sumatriptan formulations (i.e., oral, nasal, subcutaneous) may be associated with limitations that can result in patients' delaying or avoiding treatment. For oral formulations, these limitations include difficulty in taking an oral medication due to the nausea and vomiting that often accompany migraine, and inconsistent absorption, whereas nasal and subcutaneous formulations may be associated with low bioavailability and an undesirable rate of
\end{abstract}

adverse events, respectively. An alternative to current formulations is transdermal drug delivery, particularly iontophoresis. Transdermal delivery has several advantages over current formulations, including avoidance of the gastrointestinal tract, controlled and sustained delivery, and convenient administration. This article reviews the in vitro, in vivo, and preclinical data supporting the use of iontophoresis for the delivery of sumatriptan, as well as the recent clinical data for Zelrix (NuPathe Inc., Conshohocken, PA), an iontophoretic sumatriptan patch currently in phase III development for the treatment of migraine. Key Words: Iontophoresis, migraine, transdermal, sumatriptan, Zelrix.

\section{INTRODUCTION}

Migraine is a common condition affecting nearly $12 \%$ of the population within the United States, with approximately $93 \%$ of migraineurs reporting some level of impairment during an attack and more than half (54\%) of those describing the impairment as severe. ${ }^{1}$ For example, in a 3-month period, $25 \%$ of migraineurs missed at least 1 day of work or school, $28 \%$ reported that their work or school productivity was reduced by at least half, $48 \%$ could not do typical household work, and $29 \%$ missed a family or social function. ${ }^{1}$ This is likely because migraine is a multi-symptom disorder that may include not only severe headache pain but also frequently nausea (e.g., reported in one survey to occur in a majority of episodes by $56 \%$ of respondents who have ever had nausea, in every episode by $32 \%$, and at least once by $92 \%),{ }^{2}$ and also vomiting (32\% reporting the occurrence in a majority of episodes, $13 \%$ in every episode, and $68 \%$ at least once), ${ }^{2}$ among other symptoms.

Address correspondence and reprint requests to: Mark W. Pierce, M.D., Ph.D., NuPathe Inc., 227 Washington St., Suite 200, Conshohocken, PA 19428. E-mail: mpierce@ nupathe.com.
The American Academy of Neurology guidelines indicate triptans as an effective and relatively safe initial treatment choice for patients with moderate to severe migraines. ${ }^{3}$ Among these are almotriptan, eletriptan, frovatriptan, naratriptan, rizatriptan, sumatriptan, and zolmitriptan. Triptans are believed to work by binding agonistically to serotonin receptors, primarily the $5-\mathrm{HT}_{1 \mathrm{~B}}$ and $5-\mathrm{HT}_{1 \mathrm{D}}$ receptors. ${ }^{4}$ These are expressed in the smooth muscle cells of the intracranial blood vessels and the trigeminal nerve fibers, respectively, and their activation is believed to produce vasoconstriction and block cranial vasodilatation, thereby leading to pain relief. ${ }^{4,5}$

Sumatriptan is the most frequently prescribed migraine therapy in the United States ${ }^{5}$ and is available in oral, nasal, and subcutaneous formulations. Unfortunately, each of these formulations may be associated with limitations that can result in patients' delaying or avoiding treatment. One of the primary issues with oral sumatriptan is related to nausea and vomiting associated with a migraine episode. Thirty-one percent of patients who previously experienced nausea and $42 \%$ of those who previously experienced vomiting stated that these symptoms interfered with taking oral migraine medication. ${ }^{2}$ Furthermore, because of incomplete absorption and presystemic metabolism, oral sumatriptan is also 


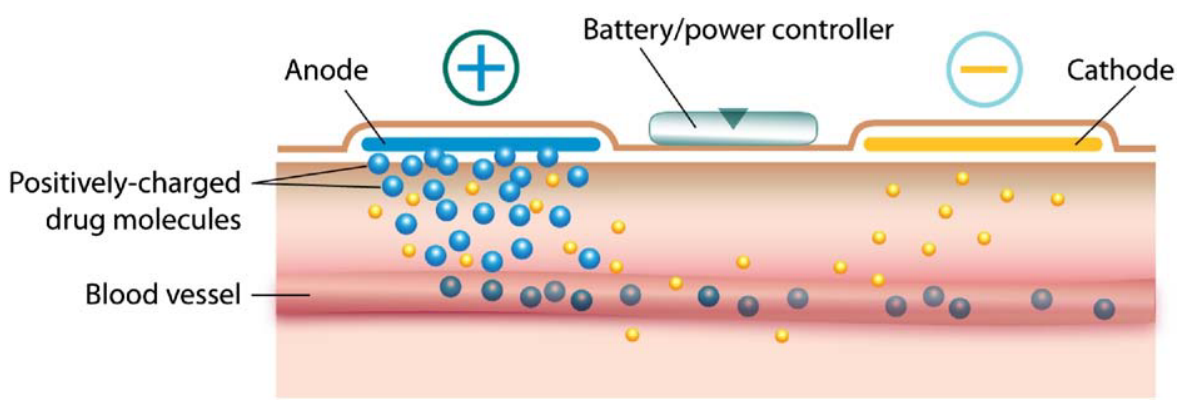

FIG. 1. Schematic of iontophoresis.

associated with low bioavailability $(\sim 15 \%){ }^{6}$ Not only does intranasal sumatriptan have a low bioavailability similar to oral sumatriptan $(\sim 17 \%)$, but as many as 1 in 4 users have reported a bad or unusual taste depending on the dose (25\% of patients with $20-\mathrm{mg}$ dose).$^{7}$ Finally, although subcutaneous sumatriptan has a much higher bioavailability $\left(\sim 97 \%^{8}\right)$, injections are often the least attractive option for patients and may be associated with an undesirable rate of adverse events (primarily injection site reactions [59\% of patients] and atypical sensations $[42 \% \text { of patients }]^{8}$ ).

An alternative to these methods is transdermal delivery, a well-established route of administration with benefits including avoidance of the gastrointestinal tract and first pass metabolism, sustained and controlled delivery, and convenient administration. ${ }^{9}$ This article will review the history of transdermal delivery of sumatriptan, with a particular focus on iontophoretic sumatriptan as an emerging option for migraineurs.

\section{PASSIVE VERSUS ACTIVE TRANSDERMAL DELIVERY}

Perhaps the most familiar means of transdermal drug delivery is through passive methods, such as ointments and passive patches (e.g., nicotine patches). The main restriction with passive delivery is the barrier property of the skin (stratum corneum), which typically limits delivery to low-molecular weight drugs that are lipophilic and effective at low doses. ${ }^{10}$ Although various attempts have been made to increase permeability (e.g., the inclusion of chemical enhancers), none of these options fundamentally change the properties of the skin, and therefore drug delivery is still limited. ${ }^{9}$

Active methods are those that engage an external energy source to help drive the drug across the skin, thereby facilitating delivery of smaller, lipophilic molecules, while also allowing larger charged and hydrophilic molecules to be transdermally delivered. Methods of active transdermal delivery include electrical (e.g., iontophoresis, electroporation) and mechanical (e.g., microneedle, abrasion), as well as others (e.g., ultrasound, magnetophoresis). ${ }^{9}$ Among the active methods, ionto- phoresis has the most application, with several iontophoretic devices already Food and Drug Administration approved and marketed (e.g., fentanyl, lidocaine) or in clinical development (e.g., acyclovir). ${ }^{10}$ An iontophoretic device is worn on the skin and may consist of an anode electrode $(+)$ in a reservoir containing positively charged drug in solution, a cathode electrode (-) in a reservoir containing a negatively charged salt solution, an electronic device to control a mild electrical current, and a power supply (FIG. 1). Once applied, the electrical current carries the positively charged drug molecules from the patch into the skin and across the stratum corneum, where it is rapidly absorbed and distributed systemically.

\section{PASSIVE TRANSDERMAL DELIVERY OF SUMATRIPTAN: IN VITRO MODEL SYSTEMS}

As previously mentioned, triptans are the most widely prescribed migraine therapies, and therefore the focus of the vast majority of published research on transdermal migraine therapy. Sumatriptan was the first triptan synthesized and is the primary triptan investigated for transdermal delivery. Initially, an in vitro standard diffusion model system was used to characterize the passive diffusion of sumatriptan and the effect of a variety of chemical enhancers. ${ }^{11}$ The model system consisted of two chambers (one with sumatriptan solution in an isotonic buffer [donor chamber] and one with isotonic buffer only [receptor chamber]) separated by porcine skin. Passive transfer was assessed by measuring the amount of sumatriptan in the receptor chamber at regular intervals using a previously validated high-performance liquid chromatography method. ${ }^{11,12}$ Several chemical enhancers were investigated, including ethanol; polyethylene glycol 600 (PEG600); Span 20 (sorbitan monolaurate); oleic acid, (-)- $\alpha$-bisabolol; 1,8cineole (eucalyptol); and R-(+)-limonene. The results demonstrated that sumatriptan could permeate the skin when enhanced by treatment with ethanol; Span 20; PEG600; oleic acid; 1,8-cineole; and R-(+)-limonene. However, it was unclear if therapeutic concentrations could be achieved.

Once it was established that sumatriptan could be delivered transdermally, additional in vitro experiments investigated the use of a bioadhesive patch ${ }^{13}$ and various 
patch substrates ${ }^{14}$ to assist with drug delivery. One of these studies examined the use of a patch made of polyvinyl alcohol (PVA), Plastoid E 35H (Rhom GmbH \& Co. KG, Darmstadt, Germany), and sorbitol, with and without Transcutol (Gattefossé, Gennevilliers Cedex, France) or PEG600 to deliver sumatriptan. ${ }^{13}$ The patches were placed on a portion of rabbit ear skin and diffusion was assessed via high-performance liquid chromatography similar to the previously described study. The total amount of sumatriptan that diffused from the patch was similar to that of sumatriptan solution, but with a higher rate of diffusion (flux) in the first 5 h. ${ }^{13}$

The previously described patch consisted mainly of PVA ( $\sim 60 \%$ dry). ${ }^{13}$ A subsequent study assessed a variety of other polymers for use in a transdermal sumatriptan system. ${ }^{14}$ Specifically, Balaguer-Fernández et al. ${ }^{14}$ examined methyl cellulose (MC), polyvinyl pyrrolidone (PVP), and a PVP-PVA mixture. The patches were developed and assessed in a fashion similar to the previous patch study, ${ }^{13}$ but with Azone (Netqem, Durham, NC) used as an enhancer. The amount of sumatriptan delivered was highest with the MC-Azone patch, with a significantly $(p<0.05)$ higher steady-state flux compared with PVP and PVP-PVA formulations. ${ }^{14}$ Based on their results, the authors concluded that to reach the plasma concentrations of sumatriptan observed with subcutaneous injection (maximum, $72 \mathrm{ng} / \mathrm{mL}$ ), the minimum surface area of a passive transdermal patch using the most effective formulation (MC-Azone) would be at least $293 \mathrm{~cm}^{2}$. Because this is not realistic, it was clear that delivery of therapeutic quantities of sumatriptan with these passive formulations would not be feasible.

\section{IONTOPHORETIC DELIVERY OF SUMATRIPTAN: IN VITRO AND PRECLINICAL MODEL SYSTEMS}

In the same study that assessed different possible patch polymers, ${ }^{14}$ the authors also investigated the application of iontophoretic technology to the MC-Azone patch and determined that based on the observed sumatriptan transdermal flux of $284.7 \mu \mathrm{g} / \mathrm{cm}^{2} \mathrm{~h}$, an iontophoretic patch would require only a $17 \mathrm{~cm}^{2}$ surface area. ${ }^{15}$ Two additional studies of iontophoretic patches were reported. The first was an in vitro study using porcine skin that assessed the effect of current density and ionic strength. ${ }^{16}$ A cathode buffer solution of $75 \mathrm{mM}$ $\mathrm{NaCl}$, plus $20 \mathrm{mM}$ HEPES ( $N$-[2-Hydroxyethyl]piperazine- $N^{\prime}$-[2-ethanesulfonic acid]) $(\mathrm{pH}=7.4)$ and an anode solution of $14.5 \mathrm{mM}$ sumatriptan succinate in the same buffer $(\mathrm{pH}=6.5)$ were placed in separate electrode chambers, each of which covered an area of $0.9 \mathrm{~cm}^{2}$. High-performance liquid chromatography was then used to quantify the amount of sumatriptan delivered by both a $0.25 \mathrm{~mA} / \mathrm{cm}^{2}$ and $0.50 \mathrm{~mA} / \mathrm{cm}^{2}$ current density; the $0.25 \mathrm{~mA} / \mathrm{cm}^{2}$ current density was also investigated using a lower ionic strength buffer solution of $25 \mathrm{mM} \mathrm{NaCl}$. The results showed that a current density of $0.25 \mathrm{~mA} /$ $\mathrm{cm}^{2}$ and a low ionic strength buffer solution $(25 \mathrm{mM})$ provided the best conditions for transdermal delivery of sumatriptan, with transdermal flux of $1273 \pm 83 \mathrm{nM} /$ $\mathrm{cm}^{2} \mathrm{~h}$ compared with a passive delivery control, a 385fold increase. ${ }^{16}$

Based on results from a separate in vitro study, the second study examined the iontophoretic delivery of sumatriptan in vivo. ${ }^{17} \mathrm{~A}$ two-compartment iontophoretic patch containing $37 \mathrm{mg}$ of sumatriptan at $\mathrm{pH} 7.0$ was placed on each of three prepubescent female pigs. The cathode and anode surface areas were $8 \mathrm{~cm}^{2}$ each, and a biphasic current protocol was used in which step 1 (from $0-180 \mathrm{~min}$ ) had a current intensity of $0.45 \mathrm{~mA} / \mathrm{cm}^{2}$, and step 2 (from 181-360 min) had a current intensity of 0.2 $\mathrm{mA} / \mathrm{cm}^{2}$. Peak sumatriptan plasma concentrations of 90 to $100 \mathrm{ng} / \mathrm{mL}$ occurred between 90 and 180 minutes after initiation of iontophoresis. ${ }^{17}$

\section{IONTOPHORETIC DELIVERY OF SUMATRIPTAN IN MAN}

Recently, clinical studies of the safety, pharmacokinetics, and efficacy of sumatriptan delivery have been reported with NP101 (Zelrix [NuPathe Inc., Conshohocken, PA]), a single-use, disposable, transdermal patch that delivers sumatriptan via iontophoresis. A phase I randomized, single-center, single-dose, 6-period, crossover study evaluated the safety and pharmacokinetic profiles of four different prototype iontophoretic sumatriptan patches (NP101) compared with $6 \mathrm{mg}$ of subcutaneous (treatment 1 [GlaxoSmithKline]) and $50 \mathrm{mg}$ of oral sumatriptan (treatment 2, Imigran FTab [GlaxoSmithKline, United Kingdom]). ${ }^{5}$ The four prototypes included: 1) NP101.01 (1 $\mathrm{mA}$ for 1.5 hours [treatment 3]); 2) NP101.02 (0.5 mA for 3 hours [treatment 4]); 3) NP101.03 (2 mA for 3 hours [treatment 5]); and 4) NP101.04 (2 mA for 6 hours [treatment 6]). A total of eight normal subjects (aged $18-50$ year) received each of the 6 treatments with a minimum of 3 days between each dosing period. Treatments 5 and 6 provided the most favorable sumatriptan profiles. Treatment 5 yielded an area under the curve from time 0 to $24 \mathrm{~h}\left(\mathrm{AUC}_{0-24}\right)$ and maximum observed concentration $\left(\mathrm{C}_{\max }\right)$ that were $88 \%$ and $109 \%$ of the oral formulation, respectively, and $122 \%$ and $49 \%$ of the subcutaneous formulation, respectively. Values for the $\mathrm{AUC}_{0-24}$ and $\mathrm{C}_{\max }$ of treatment 6 were $187 \%$ and $131 \%$ of the oral formulation, respectively, and $259 \%$ and $59 \%$ of the subcutaneous formulation, respectively. In addition, the formulation of treatment 6 delivered sumatriptan at a rate that maintained a target sumatriptan plasma concentration of at least $10 \mathrm{ng} / \mathrm{mL}$ for greater 
than 7 hours. ${ }^{5}$ NP101 treatments were generally well tolerated, with the most common adverse events being mild to moderate skin erythema at the site of patch application. This study demonstrated in man the feasibility of well-tolerated transdermal delivery of sumatriptan via iontophoresis in sufficient quantity to be potentially effective in the treatment of migraine.

A second phase I study examined the safety and pharmacokinetic profiles of two refined NP101 patches (Zelrix I, II) and compared them to subcutaneous, oral, and nasal sumatriptan. ${ }^{18}$ This study was a single-center, open-label, single-dose, crossover study in which 25 normal subjects (aged 21-57 years) received each of five treatments: 1) sumatriptan (100 mg oral), 2) sumatriptan (6 mg subcutaneous), 3) sumatriptan (20 mg nasal spray), 4) Zelrix I patch (3 g sumatriptan gel containing $120 \mathrm{mg}$ of sumatriptan succinate delivered at $4 \mathrm{~mA}$ for $1 \mathrm{~h}$ followed by $2 \mathrm{~mA}$ for $3 \mathrm{~h}$ ), and 5) Zelrix II patch (2.6 g sumatriptan gel containing $104 \mathrm{mg}$ of sumatriptan succinate delivered at $4 \mathrm{~mA}$ for $1 \mathrm{~h}$ followed by $2 \mathrm{~mA}$ for $3 \mathrm{~h}$ ). The mean drug delivery during the 4 -h period was approximately $6.1 \mathrm{mg}$ for both Zelrix patches, and the $\mathrm{C}_{\max }$ values $(24.8 \mathrm{ng} / \mathrm{mL}$ for Zelrix I and $23.1 \mathrm{ng} / \mathrm{mL}$ for Zelrix II) fell between those for the nasal $(12.5 \mathrm{ng} / \mathrm{mL})$ and oral $(51.6 \mathrm{ng} / \mathrm{mL})$ formulations. The Zelrix patch was well tolerated with no unexpected adverse events. Of note, there were no reported atypical sensations with Zelrix (a commonly reported adverse event associated with triptan use) compared with $61 \%$ of subjects reporting atypical sensations associated with the subcutaneous formulation. This may be due to the lower $\mathrm{C}_{\max }$ associated with Zelrix relative to the subcutaneous formulation. ${ }^{18}$

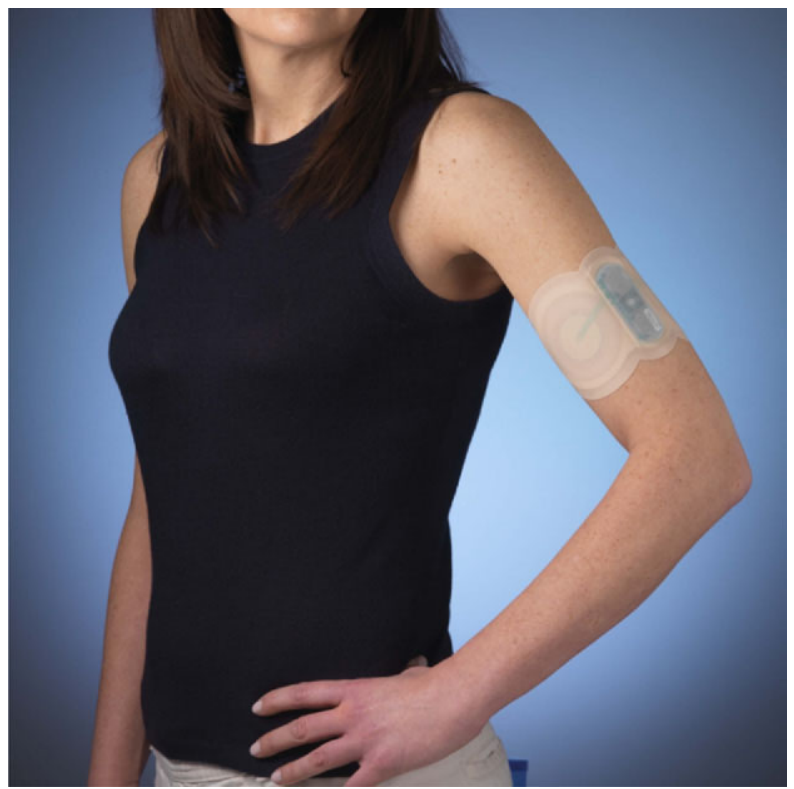

FIG. 2. Zelrix patch (NuPathe Inc., Conshohocken, PA) as worn by a patient.
The results of a randomized, double-blind, placebocontrolled phase III study of Zelrix (FIG. 2) in the treatment of acute migraine have been recently reported. ${ }^{19}$ In this trial, 530 patients meeting International Classification of Headache Disorders diagnostic criteria of migraine headache were randomly assigned 1:1 to treat a single, moderate-to-severe migraine headache with either Zelrix or a matching placebo patch. The protocol defined primary endpoint was pain free, with key secondary endpoints of photophobia free, phonophobia free, and nausea free, all at $2 \mathrm{~h}$ postpatch activation. Compared with a placebo, significantly more Zelrix patients reported freedom from headache pain (18\% Zelrix vs $9 \%$ placebo $[p=0.009])$, photophobia $(51 \%$ Zelrix vs $36 \%$ placebo $[p=0.0028])$, phonophobia (55\% Zelrix vs $39 \%$ placebo $[p=0.0002]$ ), and nausea (84\% Zelrix vs $63 \%$ placebo $[p<0.0001]$ ), whereas at the same time reporting no use of rescue medication (60\% Zelrix vs $40 \%$ placebo $[p<0.0001])$ and experiencing sustained headache pain relief for 2 to $24 \mathrm{~h}$ postpatch activation ( $34 \%$ Zelrix vs $21 \%$ placebo [ $p=0.002])$. A significantly higher proportion of patients treated with Zelrix experienced both pain relief and nausea-free endpoints at $1 \mathrm{~h}$ compared with placebo (Zelrix 29\% vs placebo $19 \%$ for pain relief [ $p=0.0135]$, and Zelrix $71 \%$ vs placebo $58 \%$ for nausea free $[p=0.0251])$. Zelrix was well tolerated, with only $2 \%$ of patients who discontinued use due to adverse events, which was the same rate as the placebo. Treatment-emergent adverse events were reported by $51 \%$ of Zelrix patients and $45 \%$ of patients receiving a placebo. These events were predominately transient application site events, with most classified as mild or moderate in intensity.

Overall, iontophoretic delivery of sumatriptan seems to have several benefits in comparison to traditional delivery methods; however, there are limitations that should be noted. Primarily, transdermal delivery may be associated with application site reactions. For example, in the previously discussed phase I and III studies of Zelrix, the most common adverse events were application site pruritus and application site pain. ${ }^{18,19}$ In both studies, these application site reactions were generally mild and resolved within 2 days. In addition, there are some limitations placed on the use of an iontophoretic patch, including restrictions on bathing and swimming once the patch is applied to the skin, and avoidance of scars, lesions, tattoos, etc, at the site of application.

\section{TRANSDERMAL DELIVERY OF OTHER TRIPTANS}

In addition to sumatriptan, transdermal delivery of rizatriptan and zolmitriptan has also been studied. The passive transdermal delivery of an elastic liposomal formulation of rizatriptan was evaluated in both in vitro and in vivo analyses. ${ }^{20}$ Compared with a solution of 
rizatriptan, the elastic liposome formulation allowed for a drug deposition that was 10-fold higher with a sustained release through 24 hours and a 3-fold increase in biologic activity, suggesting a possible future option for delivery of migraine therapy. Similar to the Zelrix patch, the iontophoretic delivery of zolmitriptan is also currently under investigation. ${ }^{21}$ In vitro and in vivo analyses demonstrated that zolmitriptan may be well suited for use in an iontophoretic device. ${ }^{21}$

\section{CONCLUSIONS}

Transdermal drug delivery is an established route of administration that has several benefits in comparison to more traditional routes, including avoidance of the gastrointestinal tract, controlled and sustained delivery, and convenient administration. ${ }^{9}$ These benefits may be attractive to migraineurs as the current oral, nasal, and subcutaneous formulations are each associated with their own limitations. Sumatriptan is one of the more commonly prescribed migraine therapies, and current research suggests that new devices, such as the Zelrix iontophoretic patch, may provide additional treatment options for migraineurs.

Acknowledgments: Editorial and medical writing support was provided by Michael Malia, PhD, at Complete Publication Solutions, LLC. This work was supported by funding from NuPathe, Inc.

\section{REFERENCES}

1. Lipton RB, Bigal ME, Diamond M, Freitag F, Reed ML, Stewart WF. Migraine prevalence, disease burden, and the need for preventive therapy. Neurology 2007;68:343-349.

2. Silberstein SD. Migraine symptoms: results of a survey of selfreported migraineurs. Headache 1995;35:387-396.

3. Silberstein SD. Practice parameter: evidence-based guidelines for migraine headache (an evidence-based review): report of the Quality Standards Subcommittee of the American Academy of Neurology. Neurology 2000;55:754-762.

4. Jhee SS, Shiovitz T, Crawford AW, Cutler NR. Pharmacokinetics and pharmacodynamics of the triptan antimigraine agents: a comparative review. Clin Pharmacokinet 2001;40:189-205.
5. Siegel SJ, O'Neill C, Dube LM, et al. A unique iontophoretic patch for optimal transdermal delivery of sumatriptan. Pharm Res 2007; 24:1919-1926.

6. GlaxoSmithKline. IMITREX (sumatriptan succinate) Tablets prescribing information. 2007.

7. GlaxoSmithKline. IMITREX (sumatriptan) Nasal spray prescribing information. 2007.

8. GlaxoSmithKline. IMITREX (sumatriptan succinate) Injection prescribing information. 2008

9. Brown MB, Martin GP, Jones SA, Akomeah FK. Dermal and transdermal drug delivery systems: current and future prospects. Drug Deliv 2006;13:175-187.

10. Prausnitz MR, Langer R. Transdermal drug delivery. Nat Biotechnol 2008;26:1261-1268.

11. Femenia-Font A, Balaguer-Fernandez C, Merino V, Rodilla V, Lopez-Castellano A. Effect of chemical enhancers on the in vitro percutaneous absorption of sumatriptan succinate. Eur J Pharm Biopharm 2005;61:50-55.

12. Femenia-Font A, Merino V, Rodilla V, Lopez-Castellano A. Highperformance liquid chromatographic determination of sumatriptan after in vitro transdermal diffusion studies. J Pharm Biomed Anal 2005;37:621-626.

13. Femenia-Font A, Padula C, Marra F, et al. Bioadhesive monolayer film for the in vitro transdermal delivery of sumatriptan. J Pharm Sci 2006;95:1561-1569.

14. Balaguer-Fernandez C, Femenia-Font A, Del Rio-Sancho S, Merino V, Lopez-Castellano A. Sumatriptan succinate transdermal delivery systems for the treatment of migraine. J Pharm Sci 2008; 97:2102-2109.

15. Femenia-Font A, Balaguer-Fernandez C, Merino V, Lopez-Castellano A. Combination strategies for enhancing transdermal absorption of sumatriptan through skin. Int J Pharm 2006;323:125-130.

16. Femenia-Font A, Balaguer-Fernandez C, Merino V, Lopez-Castellano A. Iontophoretic transdermal delivery of sumatriptan: effect of current density and ionic strength. J Pharm Sci 2005;94:21832186.

17. Patel SR, Zhong H, Sharma A, Kalia YN. In vitro and in vivo evaluation of the transdermal iontophoretic delivery of sumatriptan succinate. Eur J Pharm Biopharm 2007;66:296-301.

18. Pierce M, Marbury T, O'Neill C, Siegel S, Du W, Sebree T. Zelrix: a novel transdermal formulation of sumatriptan. Headache 2009; 49:817-825.

19. Goldstein J, Pugach N, Smith T, Nett R, Angelov A, Pierce M. Acute anti-migraine efficacy and tolerability of Zelrix, a novel iontophoretic transdermal patch of sumatriptan. Cephalalgia 2009; 29(Suppl 1):20.

20. Garg T, Jain S, Singh HP, Sharma A, Tiwary AK. Elastic liposomal formulation for sustained delivery of antimigraine drug: in vitro characterization and biological evaluation. Drug Dev Ind Pharm 2008;34:1100-1110.

21. Patel SR, Zhong H, Sharma A, Kalia YN. Controlled non-invasive transdermal iontophoretic delivery of zolmitriptan hydrochloride in vitro and in vivo. Eur J Pharm Biopharm 2009;72:304-309. 